\title{
A case of eosinophilic granulomatosis with polyangiitis complicated with a similar condition to lgG4 related lung disease
}

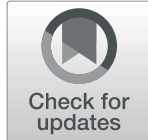

Li Zhou ${ }^{1,2,3+}$, Fen Cao ${ }^{1,2,3+}$, Songqing Fan ${ }^{4}$, Ping Chen ${ }^{1,2,3}$, Shuizi Ding ${ }^{1,2,3}$, Guiqian Liu ${ }^{1,2,3}$ and Ruoyun Ouyang ${ }^{1,2,3^{*}}$

\begin{abstract}
Background: Atypical manifestations, such as elevated serum immunoglobulin-G4 (IgG4) and extra-pulmonary lgG4 positive plasmacyte infiltration, have been described in patients with eosinophilic granulomatosis with polyangiitis (EGPA), such complicated situation might not be readily differentiated from lgG4-related disease.

Case presentation: Here, we report an interesting and rare case of EGPA in a 41 year-old male with negative anti-neutrophil cytoplasmic antibodies (ANCAs), which showed abundant pulmonary IgG4-positive plasma cells infiltration and markedly elevated serum lgG4 levels without extra-pulmonary lesions of IgG4-related disease. The clinical characteristics hesitated us whether the diagnosis as EGPA overlapping with IgG4-related lung disease should be concluded because of the absence of storiform fibrosis and obliterative phlebitis with lymphoplasmacytic infiltration. The patient's systemic symptoms, pulmonary lesions, blood eosinophils count / percentage, and serum IgG4 levels were significantly improved with immunosuppressive therapy.
\end{abstract}

Conclusions: We consider that the overlapping pathogenesis exists in the disease course of EGPA and lgG4-related disease, which may lead to interaction during the course of the diseases. And the utilization of diagnostic criteria for IgG4-related lung disease with the careful differentiation is needed in such cases.

Keywords: EGPA, IgG4-RD, Elevated serum IgG4, IgG4 positive plasma cell, Pathology of lung tissue

\section{Background}

Eosinophilic granulomatosis with polyangiitis(EGPA), previously called Churg-Straus syndrome, is a rare systematic disorder histopathologically characterized with eosinophilic infiltration, extravascular granulomas and necrotizing vasculitis predominantly affecting small to medium-sized vessels [1]. Recent research described the epidemiologic and demographic features of EGPA which showed a prevalence rates of two to 22.3 per million and the annual incidence rates of 0.5-3.7 per million and incidence peak occurred at the age of 30 to 40 or 55 to 64 year-old [2]. The clinical manifestations of EGPA are involved with severe asthma, allergic

\footnotetext{
* Correspondence: ouyangruoyun@csu.edu.cn

${ }^{+} \mathrm{Li}$ Zhou and Fen Cao contributed equally to this work.

'Department of Pulmonary and Critical Care Medicine, The Second Xiangya

Hospital, Central South University, 139 Renmin Middle Road, Changsha

410011, Hunan, China

${ }^{2}$ Research Unit of Respiratory Disease, Central South University, 139 Renmin

Middle Road, Changsha 410011, Hunan, China

Full list of author information is available at the end of the article
}

rhinitis, blood and tissue eosinophilia with cardiac, gastrointestinal, skin, renal involvement and peripheral neuropathy. And EGPA is classically considered as a Th2-mediated disease and can be subclassified as antineutrophil cytoplasmic antibodies (ANCAs) positive, which are only found in $30-40 \%$ patients with EGPA, and ANCA-negative EGPA [3, 4]. However, the accurate diagnosis of EGPA is often difficult, because of the similar or overlapping clinical manifestations to chronic eosinophilic pneumonia, hypereosinophilic syndrome, other primary systemic vasculitis, and hyperimmunoglobulin G4 syndrome [5]. IgG4-related disease (IgG4-RD) is a novel systemic immune-mediated fibroinflammatory condition involving multiple organs, and characterized by markedly increased serum IgG4 level, lymphoplasmacytic infiltration with abundant IgG4-positive plasmacytes, storiform fibrosis and obliterative phlebitis [6]. However, clinical data found increased level of serum IgG4 and/or elevated serum IgG4/IgG ratio in patients with active EGPA. And increases in IgG4

(c) The Author(s). 2019 Open Access This article is distributed under the terms of the Creative Commons Attribution 4.0 International License (http://creativecommons.org/licenses/by/4.0/), which permits unrestricted use, distribution, and 
positive plasma cells were also found in the tissue biopsies from patients with EGPA [5]. Similarity between these two diseases often causes the diagnostic dilemma to differentiate them. Herein, we report a case of EGPA patient with a pathological condition similar to IgG4related lung disease and discuss the similarity and the key difference between these two conditions. The report aims to improve the awareness of these two rare clinical diseases and prevent the diagnostic dilemma in clinical practice.

\section{Case presentation} Clinical history

A 41-year-old Chinese male was admitted to our hospital because of a 3-year history of recurrent productive cough and new onset of recurrent hemoptysis and fever for 6 months. Three years ago, the patient had been admitted to the local hospital because of cough and expectoration, and the chest computed tomography (CT) scan revealed bilateral lower lung infection. Not much improvement of respiratory symptoms had been observed, although treatments of anti-infection and antituberculosis had been administrated in turn. Besides, the patient suffered a new onset of intermittent symmetric pain of limb joints, swelling of the upper eyelid, and erythematous maculopapular rash on the dorsal surfaces of the metacarpophalangeal joint, bilateral elbow joint, and proximal interphalangeal joint. Both lower limbs developed a livedo reticularis appearance after standing for approximate $5 \mathrm{~min}$. Six months before admission, the patient experienced hemoptysis and developed a fever with maximum temperature of $38.5^{\circ} \mathrm{C}$. The frequency of redness and swelling of upper eyelids increased with and left and right eyelids alternated once a week. The new soybean-sized subcutaneous nodules with tenderness were found on the bilateral finger pulp. High potency anti-infection, anti-tuberculosis and anti-fungus treatment were given but demonstrated ineffectiveness with accelerated cough and expectoration. The patient had a 3-year history of sinusitis and family history of asthma. And he had no previous history of smoking and drinking.

\section{Physical and laboratory examinations}

Physical examination showed redness and swelling of right eyelid, and gastrocnemius tenderness. And fine moist rales and wheezes were heard in both lower lung fields. An erythematous maculopapular rash on the dorsal surfaces of the metacarpophalangeal joint of the right hand and dark red pigmentation on the right elbow were found. Laboratory studies revealed lightly elevated leukocyte count $\left(10.73 \times 10^{\wedge} / \mathrm{L}\right)$, and markedly increased eosinophil count and percentage $\left(1.26 \times 10^{\wedge} / \mathrm{L}\right.$, $11.7 \%)$, erythrocyte sedimentation rate $(99 \mathrm{~mm} / \mathrm{h})$ and
C-reactive protein $(167 \mathrm{mg} / \mathrm{L})$. Further laboratory studies showed increased levels of serum IgG $(30.8 \mathrm{~g} / \mathrm{L})$ and highly elevated levels of serum IgG4 $(24.5 \mathrm{~g} / \mathrm{L})$. Tests for IgE levels, complement system, Anti-nuclear antibody, antiextractable nuclear antigen antibody, dermatomyositisassociated antibodies, myeloperoxidase-ANCA, proteinase3-ANCA, anti-cardiolipin antibody, anti-cyclic citrullinated peptide antibody and rheumatoid factor were all normal. The ultrasounds of parotid gland, submandibular gland, thyroid, heart were all normal. Pulmonary function tests showed severe mixed pulmonary ventilation dysfunction, moderate pulmonary diffusion function, negative bronchodilation test. And the diurnal variation of peak expiratory flow (PEF) was more than $10 \%$ and the value of fractional exhaled nitric oxide was also high (26 ppb). A chest CT scan revealed diffuse groundglass opacities in both lungs and patches with increased density and blurred edges (Fig. 1a-b). A CT scan of the sinuses showed ethmoid sinusitis on the left side and maxillary sinusitis bilaterally. Bronchoscopy did not find any abnormalities and bronchoalveolar lavage fluid showed normal cellular counts and eosinophil count. Bone marrow puncture showed active proliferation of bone marrow cells, in which the proportion of osinophils was 12\%, and no abnormal pathological cells were observed. The genetic analyses showed normal FIP1L1/PDGFR $\alpha$ and ETV6-PDGFR $\beta$ fusion genes. Pathological examination of lung biopsy showed fibrosis, infiltration of lymphocytes, plasma cells and histocytes, and the presence of eosinophils in small vessels of bronchiolar walls. Immunohistochemistry revealed CD38+, CD138+, IgG+, and IgG4+ cells in which the ratio of IgG4+ to IgG+ plasma cells was greater than $40 \%$ and there were more than 10 IgG4+ plasma cells per high-power field (Fig. 2). The skin biopsy showed enriched infiltration in the vasculature and interstitial spaces of the whole dermis by eosinophils, neutrophils, and lymphoid tissue, along with focal fibrinoid necrosis in the reticular dermis (Fig. 3).

\section{Diagnostic basis}

There are no commonly accepted diagnostic criteria for EGPA. And the classification criteria for EGPA defined by American College of Rheumatology (ACR) was widely used by clinician [7]. It is composed of six criteria for EGPA: (1) asthma, (2) eosinophilia $>10 \%$, (3) neuropathy(mono- or poly-neuropathy), (4) nonfixed pulmonary infiltrates, (5)paranasal sinus abnormality, (6) extravascular eosinophil infiltration on biopsy finding. When four or more of the above six criteria are present, vasculitis can be classified as EGPA with a sensitivity of $85 \%$ and specificity of 99.7\%. Our patient had typical symptoms (cough, 

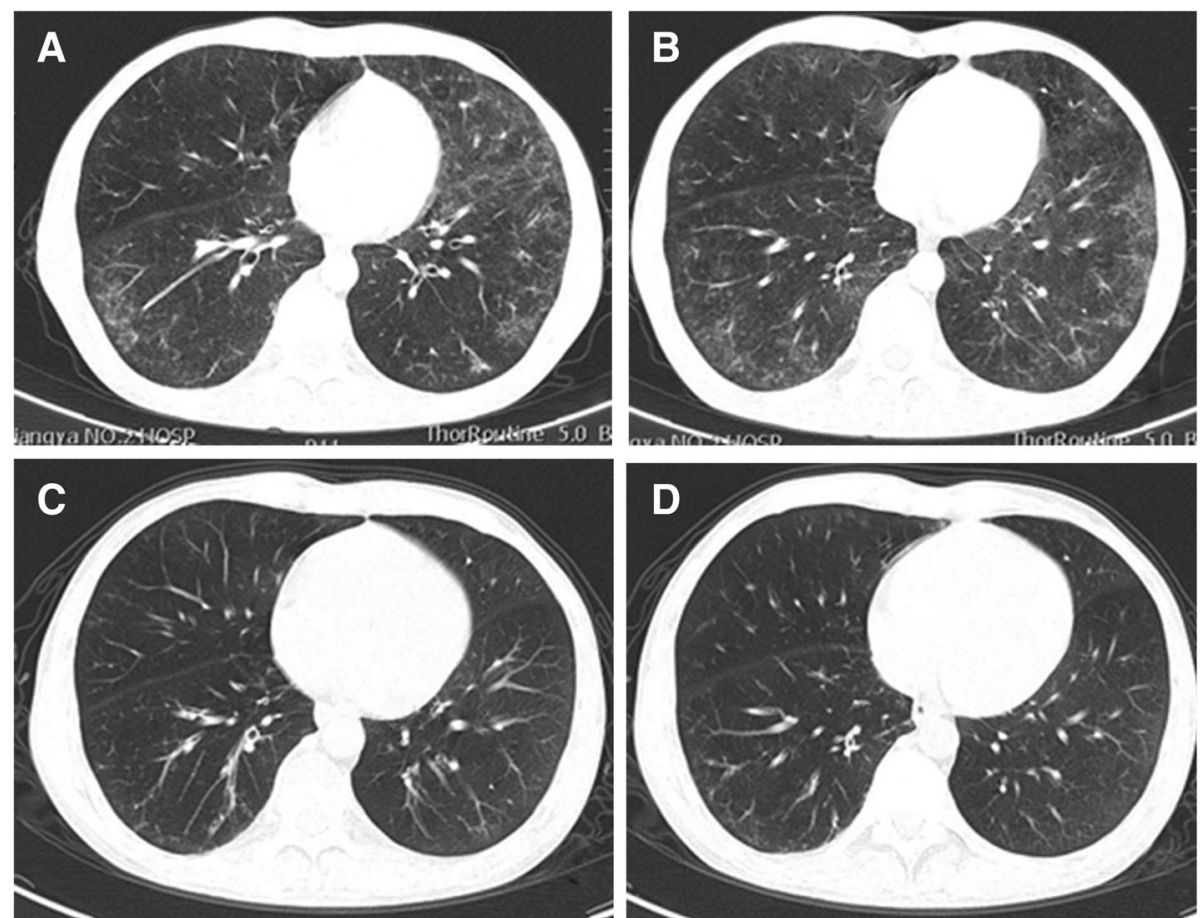

Fig. 1 a-b Chest $C T$ scan on admission showed increased bilateral pulmonary texturesand local mosaic perfusion. And diffuse ground-glass opacities and patches with increased density and blurry edges presented in both lungs. c-d One month after immunosuppressive therapy, chest CT scan showed absorption and marked improvement of pulmonary lesions
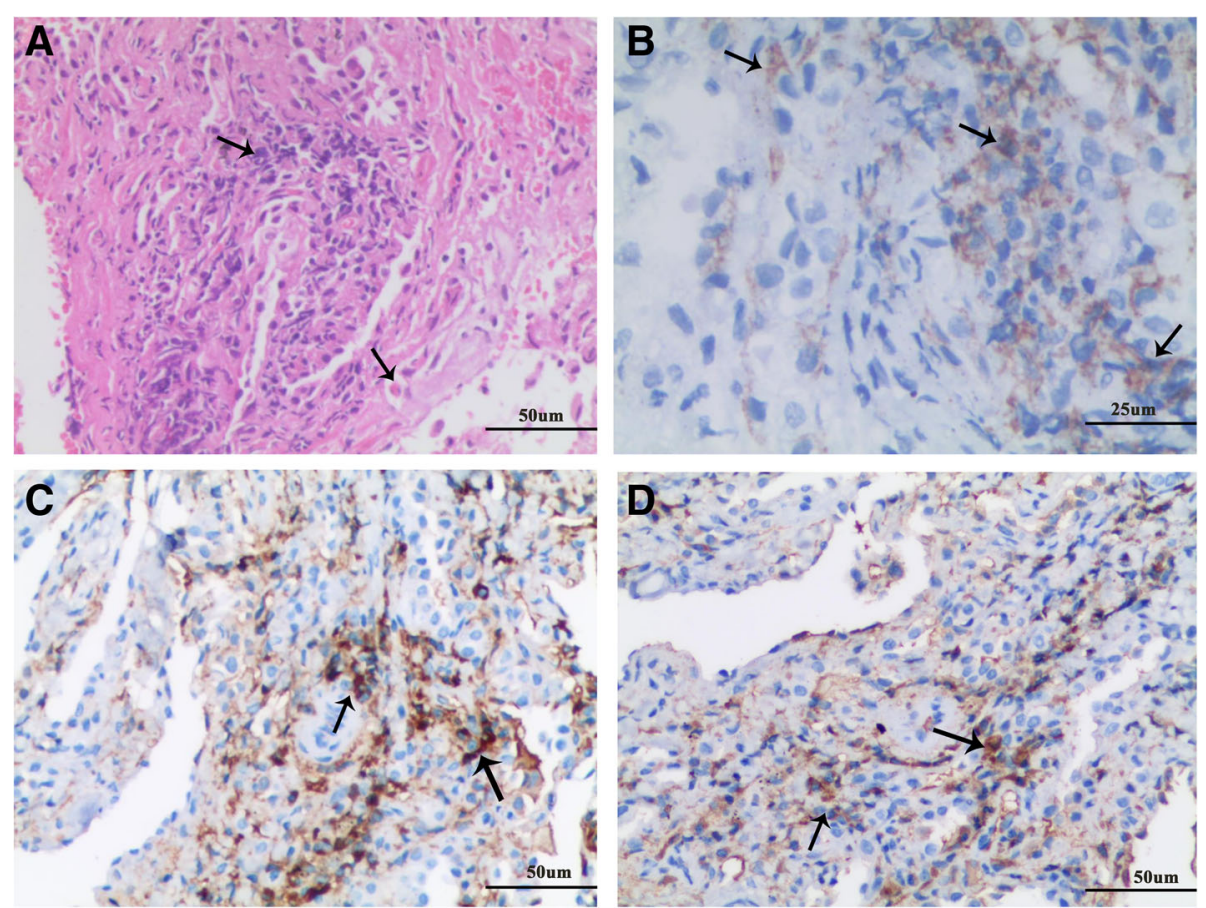

Fig. 2 Lung biopsy: a Arrows indicate infiltration of lymphocytes, histiocytes, and eosinophils in the lung tissue (HE staining; magnification 200x). b Immunohistochemistry study for CD138 showed positive CD138 cells-plasma cells (arrow; DAB staining; magnification 400x). c Immunohistochemical examination with IgG-specific antibody for specimens from lung biopsy, showing positive infiltration of lgG plasma cells (arrow; magnification 400x); d Immunohistochemical examination with IgG4-specific antibody for specimens from lung biopsy, indicating enriched lgG4+ plasma cell infiltration. (arrow; magnification 200X). HE, hematoxylin-eosin; as indicated by the arrows 

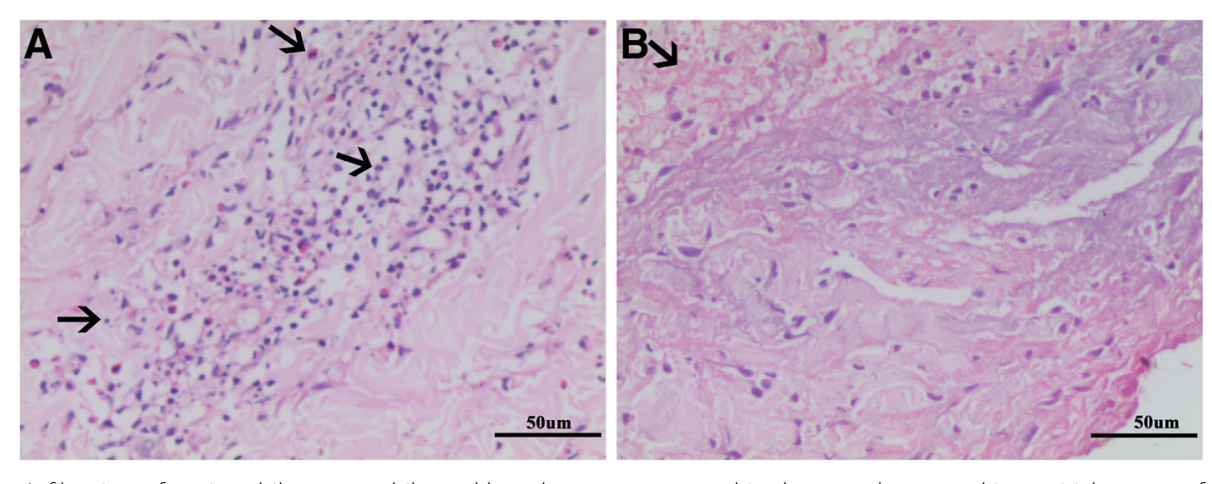

Fig. 3 Skin biopsy: a Infiltration of eosinophils, neutrophils, and lymphocytes presented in the vasculature and interstitial spaces of the dermal layer (arrows; HE staining; magnification 200x); b The arrow showed focal fibrinoid necrosis in the reticular dermis (HE staining; magnification 200x)

shortness of breath) and physical sign (wheezes in lower lungs) of asthma for more than 3 years. And the average daily diurnal PEF variability was more than $10 \%$. Besides, the patient had the family history of asthma plus with high value of fractional exhaled nitric oxide. Therefore, the diagnosis of asthma was established. The possible cause for the negative bronchodilation test is that the patient was taking inhaled budesonide-formoterol (inhaled corticosteroids-longacting beta ${ }_{2}$-agonist drug, ICS-LABA) medication to relieve shortness of breath before admission and during hospitalization. Furthermore, the percentage of blood eosinophil was $11.7 \%$, which was more than $10 \%$. The patient also had a 3-year history of sinusitis, which was also confirmed by CT scan of sinuses. The chest CT scan showed non-fixed pulmonary infiltration, with multiple patches in both lungs and groundglass opacities. Moreover, the skin biopsy showed enriched eosinophil infiltration in the vessels and interstitial spaces of the dermis. Therefore, the patient was diagnosed as EGPA with following diagnostic basis: asthma, peripheral blood eosinophilia (>10\%), paranasal sinusitis, transient pulmonary infiltration and eosinophil infiltration of the skin.

\section{Treatment}

We discontinued all of the patient's medications which had been prescribed before admission. Immunosuppressive treatment with intravenous methylprednisolone (80 $\mathrm{mg} \times 3 \mathrm{~d}, 40 \mathrm{mg} \times 3 \mathrm{~d}$ )was started and followed by oral methylprednisolone $(40 \mathrm{mg} / \mathrm{d})$. Seven days later, respiratory symptoms, like cough and expectoration, were improved. Hemoptysis, fever, redness and swelling of eyelid and polyarthralgia were all completely disappeared. The follow-up laboratory tests showed reduced eosinophils count and percentage $\left(0.07 \times 10^{\wedge 9} / \mathrm{L}, 0.40 \%\right)$, and serum IgG4 level $(16.6 \mathrm{~g} / \mathrm{L})$. After discharge, treatment of oral methylprednisolone with $35 \mathrm{mg} / \mathrm{d}$ and intravenous cyclophosphamide ( $0.4 \mathrm{~g}$ per 2 week) were both administrated.
One month later, cough, expectoration, maculopapular rash, subcutaneous nodules, reticulated blemishes were all completely disappeared. Gastrocnemius tenderness was also reduced. The follow-up laboratory tests showed further decreased eosinophils count and percentage $\left(0.01 \times 10^{\wedge} / \mathrm{L}, 0.10 \%\right)$ and serum IgG4 level $(7.41 \mathrm{~g} / \mathrm{L})$. The follow-up thoracic CT scan showed that bilateral lung lesions were also significantly improved (Fig. 1c-d). Summary of the patient's symptoms and corresponding treatment was described in the Table 1.

\section{Discussion and conclusion}

The present report shows a rare case of EGPA complicated with marked elevation of serum IgG4 concentration and abundant IgG4+ plasmacytes infiltration in the lung tissue, which lead to the a diagnostic difficulty regarding whether IgG4-related lung disease should be concluded to this patient. The updated comprehensive diagnostic criteria for IgG4-RD includes: (1)clinical manifestation with typical diffuse/localized swelling or masses in single/multiple organs; (2)elevated serum IgG4 concentrations $(>1.35 \mathrm{~g} / \mathrm{L})$; (3) characteristic histopathological examination showing significant lymphocytes and plasmacytes infiltrate, fibrosis and enriched infiltration of IgG4+ plasmacytes with ratio of $\operatorname{IgG4}+$ to IgG+ cells $>40 \%$ and $>10$ IgG4+ plasma cells per highpower field [8]. Definite IgG4-RD was established by suggestive organ involvement, combination with highly increased serum IgG4 levels and characteristic histological manifestations. However, storiform fibrosis and obliterative phlebitis with lymphoplasmacytic infiltration, two specific histopathological features in the patients with IgG4-RD, and involvement of other organs (lacrimal or salivary gland, lymph nodes, pancreas or kidney etc.) were absent in our case, which hesitated us whether it is reasonable to give a diagnosis of IgG4-RD simultaneously. No organ-specific diagnostic criteria has been established for IgG4-related lung disease. Markedly elevated serum IgG4 level and enriched infiltration of 
Table 1 Summary of the patient's symptoms, applied treatment and improvement rating

\begin{tabular}{|c|c|c|c|}
\hline Timeline & Symptoms & Treatment & Improvement rating \\
\hline 2013 & Productive cough & Repeated anti-infection therapy & Re-occurrence \& exacerbation \\
\hline March, 2016 & $\begin{array}{l}\text { Exacerbation in cough, dyspnea, } \\
\text { hemoptysis, recurrent fever, redness, } \\
\text { swelling of eyelid and polyarthralgia }\end{array}$ & $\begin{array}{l}\text { All kinds of Antibiotics therapy } \\
\text { (Piperacillin/tazobartan, cefmenoxime, } \\
\text { meropenem, levofloxacin etc.) } \\
\text { Anti-tuberculosis therapy } \\
\text { Antifungal therapy }\end{array}$ & No improvement \& exacerbation \\
\hline Sep 14-18, 2016 & $\begin{array}{l}\text { Exacerbation in cough, dyspnea, } \\
\text { hemoptysis, recurrent fever, redness, } \\
\text { swelling of eyelid and polyarthralgia }\end{array}$ & $\begin{array}{l}\text { Piperacillin/tazobartan, Amikacin, } \\
\text { roxithromycin }\end{array}$ & No improvement \\
\hline Sep 19-30, 2016 & $\begin{array}{l}\text { Productive cough, recurrent fever, } \\
\text { hemoptysis, dyspnea, swelling of eyelid } \\
\text { and polyarthralgia }\end{array}$ & $\begin{array}{l}\text { cefoperazone/sulbactam, levofloxacin, } \\
\text { azithromycin }\end{array}$ & No improvement \\
\hline Oct 1-7, 2016 & $\begin{array}{l}\text { Increased productive cough, recurrent } \\
\text { fever, swelling of eyelid and } \\
\text { polyarthralgia }\end{array}$ & Levofloxacin, roxithromycin & No improvement \\
\hline Oct 8-12,2016 & $\begin{array}{l}\text { Productive cough, subxiphoid pain, sore } \\
\text { throat, dyspnea, recurrent fever, swelling } \\
\text { of eyelid and polyarthralgia }\end{array}$ & Ceftazidime, amikacin, roxithromycin & No improvement \\
\hline Oct 12-18,2016 & $\begin{array}{l}\text { Productive cough, subxiphoid pain, sore } \\
\text { throat, dyspnea, recurrent fever, swelling } \\
\text { of eyelid and polyarthralgia }\end{array}$ & intravenous methylprednisolone & $\begin{array}{l}\text { Significant improvement or } \\
\text { disappearance in symptoms and } \\
\text { laboratory examinations }\end{array}$ \\
\hline From Oct 19, 2016 & Some cough and expectoration & $\begin{array}{l}\text { Oral methylprednisolone \& intravenous } \\
\text { cyclophosphamide }\end{array}$ & $\begin{array}{l}\text { Further obvious improvement } \\
\text { and disappearance in symptoms } \\
\text { and laboratory examinations }\end{array}$ \\
\hline
\end{tabular}

IgG4+ plasma cells in this patient may implicate the diagnosis for IgG4-RD, but we could not confirm whether eyelids swelling and lung lesions (diffuse ground glass, patchy density) were resulted from IgG4-RD.

In recent years, elevation of serum IgG4 level and/or IgG4+ plasma cells infiltrate were found in multiple lung diseases, such as pulmonary malignancy, granulomatosis with polyangiitis, EGPA, interstitial pneumonias, multicentric castleman's disease [6]. A high ratio of IgG4 to IgG positive plasma cell $(>40 \%)$ and $>50$ IgG4+ plasma cells in a high-power filed without extra-thoracic manifestation of IgG4-RD have been reported in 5 patients with surgical lung biopsy-proved idiopathic interstitial pneumonia in a retrospective study [9]. EGPA may be also accompanied by elevated serum IgG4 levels. Several studies have showed elevated serum IgG4 level $[10,11]$ and/or infiltration of IgG4-positive plasma cell in the tissue biopsy, two crucial features in patients with IgG4RD, among EGPA patients [12, 13]. Nobuhiro et al. described a EGPA case with a similar clinical condition of IgG4-related kidney disease, in which the patient was involved with a high serum IgG4 level, salivary gland swelling, tubulointerstitial nephropathy and infiltrate of IgG4 positive plasmacytes in the renal tissue, although the ratio of IgG4+ plasma cells to all IgG+ plasma cells is about $10 \%$ (less than $40 \%$ ). Suguru et al. presented a case showing numerous infiltration of IgG4-positive plasma cells (about 50\%) in the right upper eyelid and high serum IgG4 concentration $(119 \mathrm{mg} / \mathrm{dl})$ during the disease course of EGPA [13]. Besides, the ratio of serum IgG4 to IgG and IgG4 levels were elevated in the disease course. Furthermore, a study found that there was no significant difference in IgG subclasses between IgG4RD and EGPA [11]. A European retrospective multicenter observational study in patients with diagnosis of ANCA-associated vasculitides and IgG4-RD concomitantly showed that lymphoplasmacytic infiltration with IgG4/IgG ratio $>40 \%$ and/or $>10 \mathrm{IgG}+$ plasma cell per high-power field was noted in the kidney, orbital mass or aorta biopsy [14]. On the other hand, studies showed that up to $50 \%$ of patients with IgG4-RD have a clinical history of allergic condition, such as asthma, sinusitis [15]. Marked eosinophilia and highly elevated IgE levels, which are features of EGPA, have also been commonly reported in patients with IgG4-RD. [16, 17] All above studies demonstrated a diagnostic dilemma and overlapped immunopathogenetic mechanisms existed in the disease course of these two diseases. Or whether IgG4RD can be developed from EGPA?

Up-regulated responses of $\mathrm{T}$ helper (Th) 2 cell and $\mathrm{T}$ regulatory (Treg) cells and their cytokines (interleukin (IL)-4, IL-5, IL-13, IL-10 and transforming growth factor beta 1) play a key role in the pathogenesis of IgG4-RD. [15] EGPA is classically considered as a Th2 cellularmediated immune-inflammatory disease, in which high levels of Th2-associated cytokines (IL-4, IL-5 and IL-13) 
are secreted and precipitates a B-cell response resulting in the production of IgE, IgG4 and ANCA and simultaneously increases expression and secretion of eotaxin-3 guiding eosinophil to the endothelium and tissues resulting in the eosinophilia and damage of organs [4]. The cytokines IL-4, IL-5, IL-13 have been demonstrated to be involved in triggering the production of IgE or generation of IgE-producing plasma cells and promoting the production of IgG4 [18]. Besides, IgG4, a Th2dependent IgG subclass, and IgE both can be induced by allergens [19]. More importantly, IgE can be switched into IgG4 under the effect of IL- 4 and IL-13, which indicates elevated levels of IgG4 in the Th2-immune responses may be associated with the increases of IgE. Above evidences seem to well explain, to some extent, why EGPA and IgG4-RD share common clinical manifestations, like asthma and allergy, markedly elevated IgG4 levels, and abundant infiltration of IgG4 positive plasma cells. And we consider whether our patient was just during the early stage of IgG4-RD. Or maybe EGPA interact with IgG4-RD and the disease course of EGPA can promote the onset of IgG4-RD due to the overlap pathogenesis.

In summary, giving a diagnosis of IgG4-RD may be challenging in such cases and requires a comprehensive consideration with clinical symptoms, laboratory examinations and histopathologic studies. We gave the patient the diagnosis of EPGA complicated with probable IgG4RD eventually. Fortunately, recommended first-line therapy for EPGA and IgG4-RD are both immunosuppressive therapy with glucocorticoids $[6,20]$. In our case, the patient received intravenous methylprednisolone and adjuvant treatment with intravenous pulses of cyclophosphamide because of involvement of multiple organs. The patient's pulmonary and systemic symptoms, pulmonary lesions, laboratory tests for blood eosinophil count and serum IgG4 levels were all significantly improved with above treatment.

In conclusion, it is the first report showing markedly elevated serum IgG4 levels and abundant infiltration of IgG4 positive plasma cells in the lung tissue simultaneously in patient with EGPA, which strongly suggest us that EGPA and IgG4-RD may share an overlapped pathogenesis in local and systemic IgG4 response. We consider that EGPA and IgG4-RD may intersect in the course of disease and, in some extent, the development of one disease can influence the onset of another disease. Further studies should be required to investigate the potential mechanism.

\section{Abbreviations}

ANCA: Antineutrophil cytoplasmic antibodies; CT: Computed tomography; EGPA: Eosinophilic granulomatosis with polyangiitis; IgG4: ImmunoglobulinG4; IgG4-RD: IgG4-related disease; IL: Interleukin

\section{Acknowledgements}

We thanks all the staff at the Department of Pulmonary and Critical Care Medicine for their help in completion of this study.

\section{Authors' contributions}

LZ was the major contributor in writing and revising the manuscript. FC and LZ made substantial contribution to collection and interpretation of the patient's clinical data. SQF was contributed to performing the examinations of immunohistochemical pathology and diagnosis for the patient. PC and RYOY were contributed to the design of the work and responsible for diagnosis and treatment of the patient. RYOY and SQF were also critically contributed to the revision of the paper. SZD was contributed to supplemental collection of clinical data of the case. GQL participated in the collection of radiological data. All authors read, reviewed and approved the final manuscript.

\section{Funding}

This study was supported by Projects of National Key Clinical Specialist Construction [Grant (2012) 650]. The funders had no role in the design, collection, analysis and interpretation of data and writing of the manuscript. If the case report is accepted for publication, the funders will cover the related costs.

\section{Availability of data and materials}

All the data discussed in the manuscript are included within this published article.

\section{Ethics approval and consent to participate}

Not applicable.

\section{Consent for publication}

Written informed consent for publication of case report was obtained from the patient.

\section{Competing interests}

The authors declare that they have no competing interests.

\section{Author details}

${ }^{1}$ Department of Pulmonary and Critical Care Medicine, The Second Xiangya Hospital, Central South University, 139 Renmin Middle Road, Changsha 410011, Hunan, China. ${ }^{2}$ Research Unit of Respiratory Disease, Central South University, 139 Renmin Middle Road, Changsha 410011, Hunan, China. ${ }^{3}$ Diagnosis and Treatment Center of Respiratory Disease, Central South University, 139 Renmin Middle Road, Changsha 410011, Hunan, China. ${ }^{4}$ Department of Pathology, The Second Xiangya Hospital, Central South University, Changsha, China.

Received: 2 May 2019 Accepted: 12 August 2019

Published online: 19 August 2019

\section{References}

1. Jennette JC, Falk RJ, Bacon PA, et al. 2012 revised international Chapel Hill consensus conference nomenclature of Vasculitides. Arthritis Rheum. 2013;65(1):1-11.

2. Gibelin A, Maldini C, Mahr A. Epidemiology and etiology of wegener granulomatosis, microscopic polyangiitis, churg-strauss syndrome and goodpasture syndrome: vasculitides with frequent lung involvement. Semin Respir Crit Care Med. 2011;32(3):264-73.

3. Noth I, Strek ME, Leff AR. Churg-Strauss syndrome. Lancet. 2003;361(9357): 587-94.

4. Vaglio A, Buzio C, Zwerina J. Eosinophilic granulomatosis with polyangiitis (Churg-Strauss): state of the art. Allergy. 2013;68(3):261-73.

5. Mahr A, Moosig F, Neumann T, et al. Eosinophilic granulomatosis with polyangiitis (Churg-Strauss): evolutions in classification, etiopathogenesis, assessment and management. Curr Opin Rheumatol. 2014;26(1):16-23.

6. Khosroshahi A, Wallace ZS, Crowe JL, et al. International consensus guidance statement on the management and treatment of IgG4-related disease. Arthritis Rheumatol. 2015;67(7):1688-99.

7. Masi AT, Hunder GG, Lie JT, et al. The American College of Rheumatology 1990 criteria for the classification of Churg-Strauss syndrome (allergic granulomatosis and angiitis). Arthritis Rheum. 1990;33(8):1094-100. 
8. Umehara H, Okazaki K, Masaki Y, et al. Comprehensive diagnostic criteria for IgG4-related disease (IgG4-RD), 2011. Mod Rheumatol. 2012;22(1):21-30.

9. Ikeda S, Sekine A, Baba T, et al. Abundant immunoglobulin (lg)G4-positive plasma cells in interstitial pneumonia without extrathoracic lesions of IgG4-related disease: is this finding specific to lgG4-related lung disease? Histopathology. 2017;70(2):242-52.

10. Vaglio A, Strehl JD, Manger B, et al. IgG4 immune response in Churg-Strauss syndrome. Ann Rheum Dis. 2012;71(3):390-3.

11. Yamamoto M, Takahashi $H$, Suzuki $C$, et al. Analysis of serum lgG subclasses in Churg-Strauss syndrome--the meaning of elevated serum levels of lgG4. Intern Med. 2010;49(14):1365-70.

12. Ayuzawa N, Ubara Y, Keiichi S, et al. Churg-Strauss syndrome with a clinical condition similar to lgG4-related kidney disease: a case report. Intern Med. 2012;51(10):1233-8.

13. Sato S, Morimoto J, Oguchi Y, et al. A case of eosinophilic granulomatosis with Polyangiitis complicated with a lgG4 related disease like symptoms. Case Rep Immunol. 2018:2018:3763084.

14. Danlos FX, Rossi GM, Blockmans D, et al. Antineutrophil cytoplasmic antibody-associated vasculitides and IgG4-related disease: a new overlap syndrome. Autoimmun Rev. 2017;16(10):1036-43.

15. Vasaitis L. IgG4-related disease: a relatively new concept for clinicians. Eur J Intern Med. 2016:27:1-9.

16. Meridor K, Levy Y. Immunoglobulin G4-related disease presenting with clinical similarity to Churg-Strauss syndrome. Isr Med Assoc J. 2019;21(2):124-5.

17. Della Torre $\mathrm{E}$, Mattoo H, Mahajan VS, et al. Prevalence of atopy, eosinophilia, and IgE elevation in IgG4-related disease. Allergy. 2014;69(2):269-72.

18. Nirula A, Glaser SM, Kalled SL, et al. What is lgG4? A review of the biology of a unique immunoglobulin subtype. Curr Opin Rheumatol. 2011;23(1):119-24.

19. Wolfson AR, Hamilos DL. Recent advances in understanding and managing IgG4-related disease. F1000Res. 2017;6.

20. Groh M, Pagnoux C, Baldini C, et al. Eosinophilic granulomatosis with polyangiitis (Churg-Strauss) (EGPA) consensus task force recommendations for evaluation and management. Eur J Intern Med. 2015;26(7):545-53.

\section{Publisher's Note}

Springer Nature remains neutral with regard to jurisdictional claims in published maps and institutional affiliations.

Ready to submit your research? Choose BMC and benefit from:

- fast, convenient online submission

- thorough peer review by experienced researchers in your field

- rapid publication on acceptance

- support for research data, including large and complex data types

- gold Open Access which fosters wider collaboration and increased citations

- maximum visibility for your research: over $100 \mathrm{M}$ website views per year

At $\mathrm{BMC}$, research is always in progress.

Learn more biomedcentral.com/submissions 